\title{
C-beta energy converter efficiency modeling
}

\author{
Oleg Kuznetsov ${ }^{1, *}$, Viktor Chepurnov ${ }^{1, * *}$, Albina Gurskaya ${ }^{1, * * *}$, Mikhail Dolgopolov ${ }^{1, * * * *}$, \\ and Sali Radzhapov ${ }^{2, \dagger}$ \\ ${ }^{1}$ Samara University, Samara, Russia \\ ${ }^{2}$ Physical-Technical Institute of SPA "Physics-Sun" Tashkent, Uzbekistan
}

\begin{abstract}
To construct beta converters with maximum efficiency it is necessary to carry out the theoretical calculation in order to determine their optimal parameters - the geometry of the structure, the thickness of the deposition of the radioisotope layer, the depth and the width of the p-n junction, and others. To date, many different theoretical models and calculations methods had been proposed. There are fairly simple theoretical models based on the Bethe-Bloch formula and the calculation of the rate of generation of electron-hole pairs, and on calculations by equivalent circuits. Also, the Monte-Carlo method is used for theoretical modeling of beta converters. This paper explores beta converter optimization using the Monte-Carlo method. The purpose of the study is to conduct Monte-Carlo simulation of the beta converter to determine its optimal parameters.
\end{abstract}

\section{Introduction}

The General trend in the electronics of semiconductor structures associated with the miniaturization of equipment, has renewed interest in the study of betavoltaic effect $[1,2]$, which was discovered in the early XX-th century. Attempts to create devices for obtaining electric energy on the basis of this effect began a little later [3,4]. At the moment, such power supplies can work much longer than known batteries, and their use is assumed for autonomous operation, for example, sensors for monitoring various processes in construction, in production [5]. Also, the inclusion of such power sources is possible in new types of sensors of micro-and nanoscale autonomous operation with low energy consumption, in "smart" systems [6].

The principle of operation of a betavoltaic cell is similar to that of solar cells, where electron-hole pairs are generated in the space charge region when irradiated by photons. Hovewer, unlike photons, high energy beta electrons can create multiple electron-hole pairs. In a betavoltaic cell radiation sources are radioisotopes experiencing beta decay. To date, such isotopes as H-3, Ni-63, C-14, S-35 and semiconductor structures $\mathrm{SiC}, \mathrm{GaN}$, GaAs are considered in this area. These semiconductors have a wide band gap and high Debye temperature, which determines the resistance of the material to external influences. Among the mentioned semiconductor structures silicon carbide can be distinguished, which is suitable for operation

\footnotetext{
*e-mail: electron97@ rambler.ru

**e-mail: chvi44@yandex.ru

***e-mail: a-gurska@yandex.ru

****e-mail: mvdolg@yandex.ru

†e-mail: rsafti@mail.ru
} 
at temperatures over $400^{\circ}$ in corrosive environments, as well as in radioactive conditions [7]. In the previous work, the authors considered some issues related to the choice of radioisotopes, semiconductor structure and technology for obtaining a semiconductor structure for betavoltaics [8]. A good overview of the current state of the problem is available in [9] where, in particular, says about the physical characteristics of betapropiolactone.

A theoretical study of the efficiency of beta-decay energy conversion by a semiconductor requires the construction of a model for the generation of electron-hole pairs, taking into account the deposition/implantation thickness of the radioisotope layer, the depth and width of the p-n junction and a number of other characteristics. Among the approaches mentioned are methods of moments related to probability theory and mathematical statistics [10], methods of mathematical physics [11], estimation techniques [12]. In these methods, a series of papers on theoretical model calculations of beta-converters, in particular, on the basis of Ni63, which allows to make a conclusion about the existence of the maximum thickness of the active layer that contains a radioactive isotope $[13,14]$, otherwise, due to self-absorption of beta radiation can't get out of the "parent" layer and not 'create' electron-hole pairs in space charge region $\mathrm{p}-\mathrm{n}$ junction.

This article discusses the efficiency of the C-14 beta decay conversion in the silicon carbide structure, explores beta converter optimization using the Monte Carlo method. The purpose of the study is to conduct a Monte-Carlo simulation of a beta converter to determine its optimal parameters.

\section{Mathematical modeling of the beta converter using Monte-Carlo method}

\subsection{Beta-converter structure for modeling}

The structure of the beta converter based on C-14 was discussed by the authors earlier. Our theoretical model correlates with the experimental data on endotaxy of silicon structures. This method was developed at Samara University at the Department of Solid State Physics and Non-Equilibrium Systems [8]. The scheme of the developed beta-converter is presented in Figure 1. During the simulation, this scheme will be used.

This paper discusses the simulation of the beta-converter by the Monte-Carlo method in the GEANT4 library. The GEANT4 package will be used to implement the Monte Carlo simulation. GEANT4 is a library for the $\mathrm{C}++$ programming language, developed at CERN to simulate the passage of particles through matter [15]. In particular, GEANT4 is used to simulate processes in particle detectors at LHC and other accelerators. To begin modeling in GEANT4, one needs to create a geometry, that is, specify the geometric dimensions, coordinates and composition of all substances involved in the process. It is also necessary to list all the physical processes that will be taken into account in the simulation, for example, in this case, they are the ionization of substance, scattering at multiple scattering centers, and bremsstrahlung. GEANT4 incorporates cross sections of many physical processes, determined from various experiments. Then one needs to determine how much of the geometry is a detector, that is, it will record the particles flying through it. Finally, it is necessary to determine the source of the primary particles with which each simulation cycle will begin.

Once the necessary objects have been identified, statistical modeling begins. GEANT4 divides the particle trajectory into small sections - "steps". At each step, probabilities of physical processes are played out and the state of the particle - its energy, momentum, and other parameters - is updated. 


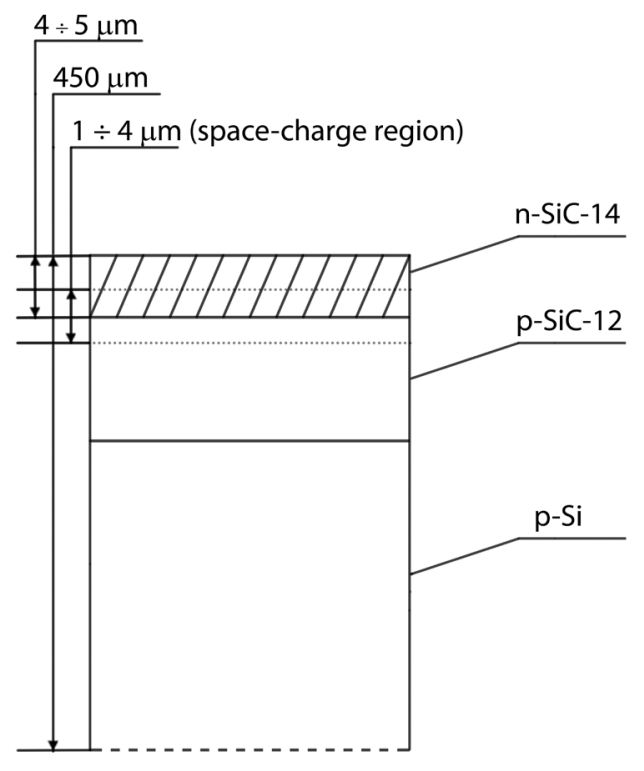

Figure 1. The structure of beta-converter.

A huge advantage of GEANT4 is the fact that it is a library for $\mathrm{C}++$. Thus, it is possible to supplement and redefine the classes built into GEANT4 and create your own, thereby adapting the package to any task.

In this paper, the Monte Carlo method and the GEANT4 package will be used to simulate the movement of primary beta electrons in a beta converter, ionization of a substance with the formation of secondary electrons, and counting the number of secondary electrons reaching the $\mathrm{p}-\mathrm{n}$ junction region. The random variables in the simulation are:

- Origin points of primary beta electrons. Beta electrons fly out during the decay of carbon14 , which occurs in the upper layer of the beta-converter, containing carbon-14. The probability of decay at a specific point is determined by the distribution of the carbon-14 concentration in this layer. Carbon-14 distribution is known from experimental data.

- Momentum directions of primary electrons. From the theory of beta decay is equally probable in all directions.

- Primary Electron Energy

Simulations will be performed $10^{7}$ times to achieve good accuracy. Since the generation rate of electron-hole pairs is the number of pairs created per unit of time, during the simulation we will assume that we have a radioactive source with an activity of $10^{7} \mathrm{~Bq}$, that is, exactly the same number of simulation cycles assumed. Thus, we can immediately calculate the values per unit time, and for the same reason these values will be directly proportional to the activity of the radioisotope.

\subsection{Calculation electron-hole pairs generation rate in the region of the $p-n$ junction}

The geometry of the model is as follows: The beta converter in GEANT4 is a volume of $1 \times 1 \times 0.1 \mathrm{~mm}$. The thickness of the top layer of $\mathrm{n}-\mathrm{SiC}$ (where $\mathrm{C}$ is $\mathrm{C}-14$ ) (green) is $5 \mu \mathrm{m}$, 


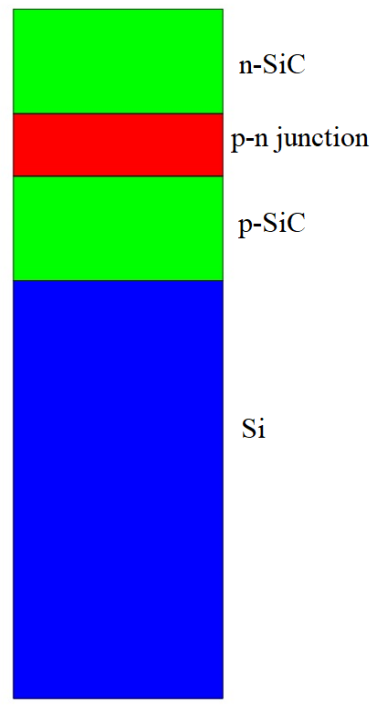

Figure 2. Model of beta-converter in GEANT4

the thickness of the Space charge region area of the $\mathrm{p}-\mathrm{n}$ junction (red) is $3 \mu \mathrm{m}$. The $\mathrm{p}-\mathrm{SiC}$ (green) layer is $5 \mu \mathrm{m}$. The rest of the volume is occupied by a silicon substrate (blue).

Simulation progress:

1. Within the $\mathrm{n}-\mathrm{SiC} 14$ region, the coordinates of the carbon 14 decay point are randomly determined.

2. The direction of the momentum of the primary beta electron and its energy are determined randomly, in accordance with the set probability densities.

3. The primary electron, passing through the sample, knocks out the secondary electrons. The trajectories of all secondary electrons are tracked in turn.

4. If the secondary electron falls into the region of the $\mathrm{p}-\mathrm{n}$ junction, this fact is noted (we increment the number of hits) and the electron is removed from consideration.

During the runing, $10^{7}$ simulations of this process were performed. The following results were obtained from the simulation results: $10^{7}$ primary beta electrons formed 6039018 electron-hole pairs in the SCR of the $\mathrm{p}-\mathrm{n}$ junction. Since $10^{7}$ primary electrons are assumed to be released per unit of time, we can obtain a formula relating the activity of a radioisotope and the rate of generation of electron-hole pairs in the $\mathrm{p}-\mathrm{n}$ transition region:

$$
G=0.6 \times A
$$

Considering specific activity of $C^{14}$

$$
A_{C^{14}}=5.20 \times 10^{18} B q
$$

And its mass in the beta converter, which is about 0.1 micro gram we get the following value for generation rate:

$$
G=3.12 \times 10^{11}
$$




\subsection{Calculation of the optimal depth of the p-n junction}

The optimal depth of the pn junction is the depth at which the largest number of electronhole pairs are formed. To calculate the optimal depth of the p-n junction in GEANT4, the model shown in Figure 4 was made. From the model from paragraph 2.1, a layer of n-SiC14

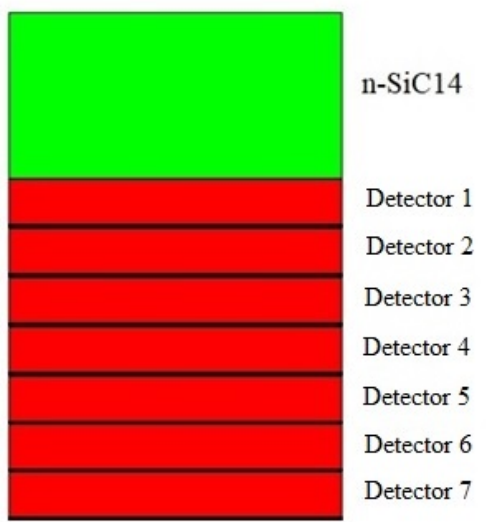

Figure 3. Model made to determine the optimal depth of the p-n junction

with carbon-14 remained. Under it were created layers of detectors with a thickness of 1 micron each. The detector number corresponds to its depth under the n-SiC14 layer. The detectors record the number of formed electron-hole pairs at a certain depth. The results of $10^{7}$ simulations of this model for the first 10 detectors are shown in Table 1.

Table 1. Result of simulations.

\begin{tabular}{|c|c|}
\hline Detector number & Amount of detected electron-hole pairs \\
\hline 1 & 2109458 \\
\hline 2 & 1679731 \\
\hline 3 & 1474314 \\
\hline 4 & 1310688 \\
\hline 5 & 1177058 \\
\hline 6 & 1061822 \\
\hline 7 & 963735 \\
\hline 8 & 879160 \\
\hline 9 & 800190 \\
\hline 10 & 727735 \\
\hline
\end{tabular}

According to the simulation results, we can conclude that the optimal depth of the $\mathrm{p}$ - $\mathrm{n}$ junction is directly below the $\mathrm{n}-\mathrm{SiC} 14$ layer. Consequently, the current beta converter design is optimal. It is important to note that out of $10^{7}$ primary electrons, about one million electron-hole pairs are created at each $1 \mu \mathrm{m}$ depth. That is, with an increase in the width of the $\mathrm{p}-\mathrm{n}$ junction by $1 \mu \mathrm{m}$, it can additionally separate another million electron-hole pairs. Thus, $1 \mu \mathrm{m}$ of the width of the SCR gives an average $10 \%$ increase in efficiency. 


\section{Summary}

Statistical modeling of the $C^{14}$ based beta-converter was performed, and the value of electronhole pairs generation rate inside the space charge region was obtained. Optimal depth of the p-n junction is determined to be right under the layer of radioisotope. We conclude that GEANT4 is a suitable framework for beta-converter modeling and determining its parameters.

Acknowledgements. Authors are grateful to organizers of The XXIV International Workshop High Energy Physics and Quantum Field Theory for the attention and opportunities of discussing results of investigations.

\section{References}

[1] Ehrenberg et al. Proceedings of the Physical Society A 64, 424 (1951)

[2] Moseley H.G.J., Harling J., Proc. R. Soc. A 88, 471 (1913)

[3] Rappaport P. I., Loferski J.J., Lindery E.G. Nucleonics 15, 99 (1957)

[4] Anno J.N., Nucl. News 3, 12 (1962)

[5] Lynch J., Loh K., Shock Vib. Dig. 38, 91 (2006)

[6] Amir H. Measurement: Journal of the International Measurement Confederation 129, 589-606 (2018)

[7] Luchinin V., Tairov Yu. Modern electronics 7, 12-15 (2009)

[8] Gurskaya A.V., Dolgopolov M.V., Chepurnov V.I., Physics of Particles and Nuclei 48, 941-944 (2017)

[9] Prelas M.A. et al. Progress in Nuclear Energy 75, 117-148 (2014)

[10] Abanin I.E. Nano- and microsystems technology 183, 4-10 (2015)

[11] A. A. Gorbatsevich et al. Journal of technical physics 61, 1053-1059 (2016)

[12] Bulyarsky S.V. et al. Physics and technology of semiconductors 1, 68-74 (2017)

[13] Nagornov Yu. S. Proceedings of higher educational institutions. Volga region. Physical and mathematical Sciences 27, 136-145 (2013)

[14] Nagornov Y.S., Murashev V.N. Semiconductors 50, 16-21 (2016)

[15] GEANT4 Documentation URL: https://geant4.web.cern.ch/ 